\title{
Numerical Simulations of Gravity Driven Reversible Reactive Flows in Homogeneous Porous Media
}

\author{
H. Alhumade ${ }^{1}$ and J. Azaiez ${ }^{2}$ \\ ${ }^{1}$ Department of Chemical Engineering, The University of Waterloo, Waterloo, ON, Canada N2L 3G1 \\ ${ }^{2}$ Department of Chemical and Petroleum Engineering, The University of Calgary, Calgary, AB, Canada T2N 1N4 \\ Correspondence should be addressed to J. Azaiez; azaiez@ucalgary.ca
}

Received 1 April 2015; Accepted 9 June 2015

Academic Editor: Junwu Wang

Copyright (C) 2015 H. Alhumade and J. Azaiez. This is an open access article distributed under the Creative Commons Attribution License, which permits unrestricted use, distribution, and reproduction in any medium, provided the original work is properly cited.

\begin{abstract}
The effect of reversibility on the instability of a miscible vertical reactive flow displacement is examined. A model, where densities and/or viscosities mismatches between the reactants and the chemical product trigger instability, is adopted. The problem is governed by the continuity equation, Darcy's law, and the convection-diffusion-reaction equations. The problem is formulated and solved numerically using a combination of the highly accurate spectral methods based on Hartley's transform and the finitedifference technique. Nonlinear simulations were carried out for a variety of parameters to analyse the effects of the reversibility of the chemical reaction on the development of the flow under different scenarios of the frontal instability. In general, faster attenuation in the development and growth of the instability is reported as the reversibility of the chemical reaction increases. However, it was observed that reversibility is capable of triggering instability for particular choices of the densities and viscosities mismatches. In addition, the effect of the reversibility in enhancing the instability was illustrated by presenting the total relative contact area between the reactants and the product.
\end{abstract}

\section{Introduction}

Instability at the interface between flowing solutions in porous media can be triggered as a result of viscosities and/or densities mismatch between the fluids. This instability develops in the form of intruding fingers and is referred to as viscous fingering or Saffman-Taylor instability in the case of viscosities mismatch or as density fingering or RayleighTaylor instability in the case of densities mismatch between the fluids [1-5].

The simultaneous variation in viscosities and densities is encountered in various applications. An analytical expression for the growth of instability in a nonreactive system with variation in viscosities and densities was derived by Bacri et al. [6]. In 1992, Rogerson and Meiburg carried out a linear stability analysis to investigate the interface of a nonreactive system with densities and viscosities mismatch in porous media where both normal and tangential velocities can be present [7]. It was reported that the growth rate of the instability was not affected by tangential velocity in immiscible displacements, unlike the miscible displacements where a stabilizing role was observed. In the same year, the same authors investigated the nonlinear evolution for the unstable modes of the same system [8], where remarkable features of instability such as diagonal fingering and secondary side-finger instabilities were observed. The effects of nonmonotonic viscosities and densities profiles on the instability of vertical nonreactive displacement processes were investigated by Manickam and Homsy by conducting nonlinear simulations of the system [9]. The authors found that a stable viscous interface between the fluids can break the symmetry of the buoyancy-driven instability by acting as a barrier against the upward growth of instability.

A simple chemical reaction, $A+B \rightarrow C$, can change the physical properties such as viscosity or density of the fluids, which might affect the fate of the displacement process. Reactive flows are encountered in many applications such as in situ oil recovery, carbon dioxide sequestration, in situ groundwater remediation and chromatographic columns, transport of contaminant, laminar combustive and exothermic reactive 
flows, natural convection heat and mass transport, and reactive porous media for biological applications [10-14]. It has been shown that a frontal instability can be purely driven by a chemical reaction, where a viscous product is generated at the interface of two reactants with smaller viscosities [15]. In a series of experiments, the effect of chemical reaction on the viscosity profile of an initially unstable system was investigated by Nagatsu et al. [16-18]. The properties of the unstable miscible reactive displacements were analyzed by a number of theoretical and numerical studies. For example, Rongy et al. [19] showed that the instability of the interface in a vertical displacement process can be influenced by buoyancy-driven convection once the densities of the species are changed by a chemical reaction even for equal diffusion coefficients and equal initial concentrations of the reactants. A related study examined the buoyancy chemically driven instability where the reaction introduces a heavier product at the initial interface and where a variation in the diffusion rates of the solutions exists [20]. For horizontal geometries, Alhumade and Azaiez carried out a linear stability analysis [21] followed by nonlinear simulations [22] of the reversible reactive flow displacements. The authors investigated quantitatively and qualitatively the effects of the chemical reversibility on the instability of the flows. Hejazi and Azaiez conducted a detailed linear stability analysis [23] followed by a nonlinear simulation [24] of miscible vertical reactive displacement processes with transverse velocity. More recently, the effects of time-dependent injections on the dynamics of reactive flows in homogeneous porous media have been investigated [25].

The instability of miscible reactive solutions under gravity force is encountered in many underground flows applications, such as in the geological storage of $\mathrm{CO}_{2}$ in addition to mixing of brine [26]. The former process involves the dissolution of carbon dioxide in the reservoir's fluid and this can be modeled by a chemical reaction [27]. A heavier solution will be introduced on top of the reservoir's lighter fluid as a result of the $\mathrm{CO}_{2}$ dissolution, which will establish instability at the fluids' interface. The reversibility of the chemical reaction plays an important role in various fields such as in situ soil remediation [28] and liquid chromatographic columns [29]. This motivates the present study aimed at analyzing the effects of chemical reversibility on the frontal instability of miscible vertical reactive displacements. In this study, a system where the variations in viscosities and densities between the reactants and the product initiate a frontal instability in the absence of injection or transverse velocities is examined.

\section{Mathematical Model}

2.1. Physical Problem. Nonlinear simulations of a twodimensional displacement process are carried out in a homogeneous reservoir, where the porosity and permeability are assumed to be constant. The displacement takes place in the vertical direction, which is referred to as the $x$-axis, while the $y$-axis is perpendicular to the direction of the flow. Furthermore, both the displacing and displaced fluids are assumed to be incompressible, Newtonian, and miscible.

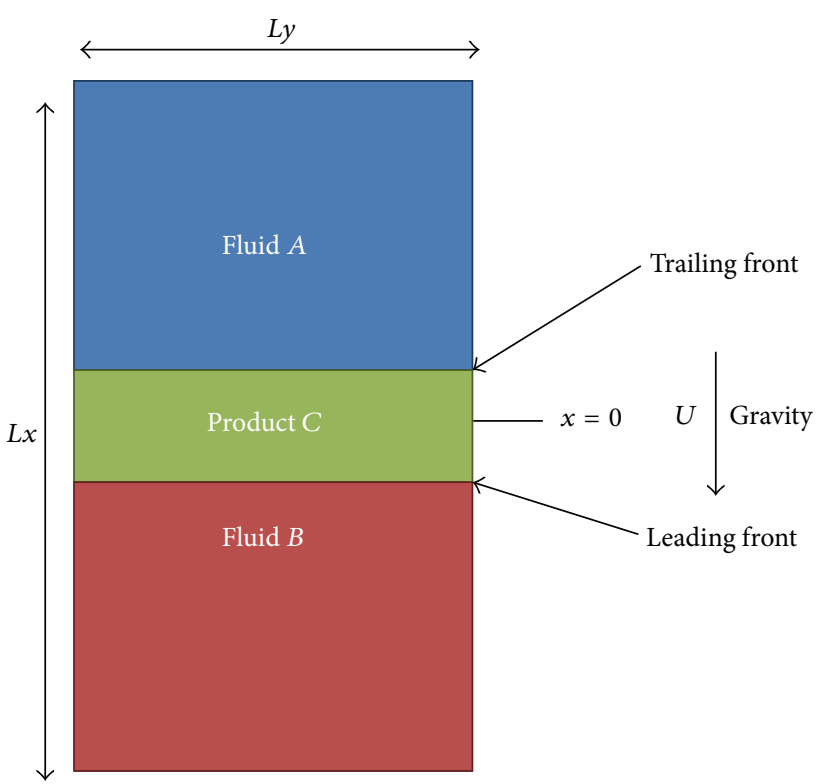

FIGURE 1: Schematic of a reactive front displacement process.

A schematic of the process is shown in Figure 1, where $L x$, $L y$, and $b$ are the length, width, and thickness of the medium, respectively. When the thickness of the medium is small compared to the other dimensions, the system corresponds to a Hele-Shaw cell, which is a common prototype for homogeneous porous media [4].

Fluid $(A)$ of viscosity $\mu_{A}$ is on top of fluid $(B)$ of viscosity $\mu_{B}$. The two fluids react to generate a chemical product $(C)$ of viscosity $\mu_{C}$ that can be different than the viscosities of both reactants. The chemical reaction is first order and can be reversible:

$$
A+B \longleftrightarrow C
$$

As time proceeds, more product accumulates at the interface between the two reactants. Figure 1 shows an idealized distribution of the two reactants $(A)$ and $(B)$ and the product $(C)$, with two fronts, one between the reactant $(A)$ and the product $(C),[A-C]$, while the other is between the reactant $(B)$ and the product $(C),[C-B]$, and they are referred to as the trailing and the leading front, respectively. It should be stressed that this is an idealization of the system and the three chemical species are actually present to a more or less degree everywhere in the region where the reaction takes place. However, this concept of a leading and trailing front will be helpful in the interpretation and explanation of the results.

2.2. Governing Equations. The flow is governed by the equations for conservation of mass, momentum (Darcy's equation), and the transport of the three chemical species:

$$
\begin{aligned}
\nabla \cdot \mathbf{u} & =0, \\
\nabla p & =-\frac{\mu}{K} \mathbf{u}+\rho \mathbf{g},
\end{aligned}
$$




$$
\begin{aligned}
& \phi \frac{\partial A}{\partial t}+\mathbf{u} \cdot \nabla A=\phi D_{A} \nabla^{2} A-k A \cdot B+k_{r} C, \\
& \phi \frac{\partial B}{\partial t}+\mathbf{u} \cdot \nabla B=\phi D_{B} \nabla^{2} B-k A \cdot B+k_{r} C, \\
& \phi \frac{\partial C}{\partial t}+\mathbf{u} \cdot \nabla C=\phi D_{C} \nabla^{2} C+k A \cdot B-k_{r} C .
\end{aligned}
$$

In the above equation, $\mathbf{u}=u \mathbf{i}+v \mathbf{j}$, where $\mathbf{i}$ and $\mathbf{j}$ are the unit vectors along $x$ and $y$, respectively, while $\nabla$ and $\nabla^{2}$ are the (nabla) differential and Laplacian operators, respectively. Furthermore, $p$ represents the pressure, $k$ the forward reaction constant, $k_{r}$ the reverse reaction constant, $\phi$ the medium porosity, $\mu$ the viscosity, $\rho$ the density, and $K$ the constant medium permeability. Here, the constant porosity and permeability are integrated in the expression of the viscosity as $\mu \phi / K$ and this expression is simply treated as $\mu$, which is referred to as the mobility or viscosity ratio. The concentrations of the two reactants and chemical product are denoted by $(A),(B)$, and $(C)$, respectively, while $D_{A}, D_{B}$, and $D_{C}$ represent their corresponding diffusion coefficients. In the present study, it will be assumed that all species have the same diffusion coefficient; that is, $D_{A}=D_{B}=D_{C}=D$. Furthermore, the maximum concentrations of both reactants are equal; that is, $a_{0}=b_{0}$. Under these conditions, the center of the reaction region remains at the initial frontal locations between the two reactants.

The above equations are expressed in a Lagrangian reference frame moving with constant velocity $U_{c h}=g|\nabla \rho| / \mu_{A}$, where $|\nabla \rho|$ is the absolute value of the difference between the densities of the displacing and displaced fluids. The equations are made nondimensional using $U_{c h}, D \phi / U_{c h}$, and $D \phi^{2} / U_{C h}{ }^{2}$ as the reference velocity, length, and time, respectively. Furthermore, the viscosity, density, pressure, and concentrations are scaled using $\mu_{A},|\nabla \rho|, \mu_{A} D \phi / K$, and $a_{0}$. The resulting dimensionless equations are

$$
\begin{gathered}
\nabla \cdot \mathbf{u}=0, \\
\nabla p=-\mu(\mathbf{u}+U \mathbf{i})+\rho \mathbf{i}, \\
\frac{\partial A}{\partial t}+\mathbf{u} \cdot \nabla A=\nabla^{2} A-D_{a} A B+D_{r} C, \\
\frac{\partial B}{\partial t}+\mathbf{u} \cdot \nabla B=\nabla^{2} B-D_{a} A B+D_{r} C, \\
\frac{\partial C}{\partial t}+\mathbf{u} \cdot \nabla C=\nabla^{2} C+D_{a} A B-D_{r} C .
\end{gathered}
$$

In the above equation $U$ is the injection velocity in the direction of the flow, which is included only to provide a complete framework model, and is assumed to be zero in the rest of this study. The Damkohler number $D_{a}=$ $k a_{0} D \phi / U_{c h}{ }^{2}$ represents the ratio between the hydrodynamic time scale and the chemical time scale, while $D_{r}=k_{r} D \phi / U_{c h}{ }^{2}$ represents a reversible Damkohler number. The ratio of these two dimensionless numbers, $\alpha=D_{r} / D_{a}$, will be referred to as the reversibility coefficient.
Following a previous related study [24], the following concentration dependent viscosity and density profiles are adopted to complete the model:

$$
\begin{aligned}
& \mu=e^{R_{b} B+R_{c} C}, \\
& \rho=G_{a} A+G_{b} B+G_{c} C .
\end{aligned}
$$

In the above equation $G_{i}=\partial \rho / \partial C_{i}$ is the density expansion coefficient of species $i$, while $R_{b}$ and $R_{c}$ are the log mobility ratios between the viscosity of $(B)$ to $(A)$ and $(C)$ to $(A)$, respectively:

$$
\begin{aligned}
& R_{b}=\ln \left(\frac{\mu_{B}}{\mu_{A}}\right), \\
& R_{C}=\ln \left(\frac{\mu_{C}}{\mu_{A}}\right) .
\end{aligned}
$$

In what follows, these log mobility ratios are simply referred to as the mobility or viscosity ratios. Furthermore, the mobility ratios at the trailing and leading fronts are referred to as

$$
\begin{aligned}
& R_{C B}=\ln \left(\frac{\mu_{B}}{\mu_{C}(C=0.5)}\right)=R_{b}-\frac{R_{C}}{2}, \\
& R_{A C}=\ln \left(\frac{\mu_{C}(C=0.5)}{\mu_{A}}\right)=\frac{R_{C}}{2} .
\end{aligned}
$$

Note that strictly positive $R_{i}(i=a, b$ or $c$ ) imply that the corresponding fronts between the fluids are viscously unstable, while negative values indicate stable fronts. Similarly for a fluid $j$ below a fluid $i$, the front is gravitationally unstable if $G_{i}>G_{j}$ and stable, otherwise.

Following previous studies [30-32], the problem is formulated in terms of vorticity $\omega$ and stream-function $\Psi$, which are related to velocity field as follows:

$$
\begin{aligned}
& u=\frac{\partial \psi}{\partial y} \\
& v=-\frac{\partial \psi}{\partial x} \\
& \omega=\frac{\partial v}{\partial x}-\frac{\partial u}{\partial y}=-\left(\frac{\partial^{2} \psi}{\partial x^{2}}+\frac{\partial^{2} \psi}{\partial y^{2}}\right) .
\end{aligned}
$$

The curl of the Darcy's law equation (8) is taken in order to eliminate the pressure, which shows that vorticity is produced by concentration gradients according to the following equation:

$$
\begin{aligned}
\omega= & R_{b}\left(\frac{\partial \psi}{\partial x} \frac{\partial B}{\partial x}+\frac{\partial \psi}{\partial y} \frac{\partial B}{\partial y}\right)+R_{c}\left(\frac{\partial \psi}{\partial x} \frac{\partial C}{\partial x}+\frac{\partial \psi}{\partial y} \frac{\partial C}{\partial y}\right) \\
& -\frac{1}{\mu}\left(G_{a} \frac{\partial A}{\partial y}+G_{b} \frac{\partial B}{\partial y}+G_{c} \frac{\partial C}{\partial y}\right) .
\end{aligned}
$$


The boundary conditions in the stream-wise direction in dimensionless form are

$$
\begin{aligned}
& u=0 ; \\
& v=0 ; \\
& B=1 ; \\
& C=0 \\
& u=0 ; \\
& v=0 ; \\
& A=0 ; \\
& B=1 ; \\
& C=0
\end{aligned}
$$

and in the transverse direction are as follows:

$$
\begin{aligned}
& (u ; v ; A ; B ; C)\left(x ;-\frac{\mathrm{Pe}}{2 A_{r}} ; t\right) \\
& \quad=(u ; v ; A ; B ; C)\left(x ; \frac{\mathrm{Pe}}{2 A_{r}} ; t\right) .
\end{aligned}
$$

In the above equations $\mathrm{Pe}=U_{c h} L_{x} / D \phi$ is the Péclet number and $A_{r}=L_{x} / L_{y}$ is the cell aspect-ratio.

2.3. Numerical Solution. The problem can be solved by splitting the variables into a base-state part and a perturbation term, where the base-state is a numerical solution of the reactive-diffusive-convective equations (4)-(6). Consider

$$
\begin{aligned}
& A(x, y, t)=A_{0}(x, y, 0)+a^{\prime}(x, y, t), \\
& B(x, y, t)=B_{0}(x, y, 0)+b^{\prime}(x, y, t), \\
& C(x, y, t)=C_{0}(x, y, 0)+c^{\prime}(x, y, t) .
\end{aligned}
$$

In the above equations, $A_{0}, B_{0}$, and $C_{0}$ refer to the solutions of the base-state while $a^{\prime}, b^{\prime}$, and $c^{\prime}$ correspond to the perturbations. The perturbation is initiated as a random noise centered at the initial interface between the reactants and that decays rapidly far from the interface [24]. The magnitude of the initial perturbation may increase, decrease, or stay constant depending on the density and viscosity mismatch between the different species. This approach where the problem is solved in terms of a perturbation results in periodic boundary conditions in both the stream-wise $(x-)$ and transverse $(y-)$ directions. Such boundary conditions allow using the highly accurate spectral methods.

Following previous studies [33-35], the Hartley pseudospectral method is used to evaluate the perturbation part of the problem. In order to implement the Hartley transform in numerical analysis, the discrete Hartley transform (DHT) and the fast Hartley transform (FHT) algorithm were introduced. The two-dimensional DHT of an arbitrary function $g(x, y, t)$ is defined as

$$
\begin{aligned}
& \hat{g}\left(k_{x}, k_{y}, t\right) \\
& =H[g(x, y, t)] \\
& =\frac{1}{\sqrt{N_{x} N_{y}}} \sum_{i=1}^{N_{x}} \sum_{j=1}^{N_{y}} g(x, y, t) \operatorname{cas}\left(\frac{2 \pi k_{x} x}{N_{x}}+\frac{2 \pi k_{y} y}{N_{y}}\right) .
\end{aligned}
$$

The above equation is a version of the DHT definition where the form of the transform and its inverse are the same. In this equation $\operatorname{cas}(x)=\cos (x)+\sin (x)$, where $k_{x}$ and $k_{y}$ represent the discrete wavenumbers while $N_{x}$ and $N_{y}$ are the number of spectral modes in the stream-wise and transverse direction, respectively.

The derivatives of a function in the Hartley transform space can be easily derived from the transfer of the function by using Hartley transform derivative theorems:

$$
\begin{aligned}
H\left[\frac{\partial}{\partial x} g(x, y, t)\right] & =-2 k_{x} \pi \hat{g}\left(-k_{x},-k_{y}, t\right), \\
H\left[\frac{\partial}{\partial y} g(x, y, t)\right] & =-2 k_{y} \pi \hat{g}\left(-k_{x},-k_{y}, t\right), \\
H\left[\frac{\partial^{2}}{\partial x^{2}} g(x, y, t)\right] & =-4 \pi^{2} k_{x}^{2} \hat{g}\left(k_{x}, k_{y}, t\right), \\
H\left[\frac{\partial^{2}}{\partial y^{2}} g(x, y, t)\right] & =-4 \pi^{2} k_{y}^{2} \hat{g}\left(k_{x}, k_{y}, t\right), \\
H\left[\frac{\partial^{2}}{\partial x \partial y} g(x, y, t)\right] & =-4 \pi^{2} k_{x} k_{y} \hat{g}\left(k_{x}, k_{y}, t\right) .
\end{aligned}
$$

This shows that the derivative terms reduce to simple algebraic expressions in the transform space. As a result, the original partial differential equations in time and space are recast into ordinary differential equations in time. The procedure for time stepping of the reactive-diffusive-convective equations in the Hartley space was based on a semi-implicit predictor-corrector method along with an operator-splitting algorithm [35].

\section{Results}

3.1. Validation and Convergence of the Numerical Code. The numerical code was validated by comparing the time evolution and the related finger structures for the case where the chemical reaction is complete $(\alpha=0)$ with those presented by Hejazi and Azaiez [24] for the nonreversible case. It was found that the dynamics of fingering were identical when the same parameters that characterize the flow were used along with the same spatial resolution and time step size. Figure 2 depicts the isosurfaces for the irreversible case at $t=1250$ for the following values of the different parameters: $R_{b}=0$, 


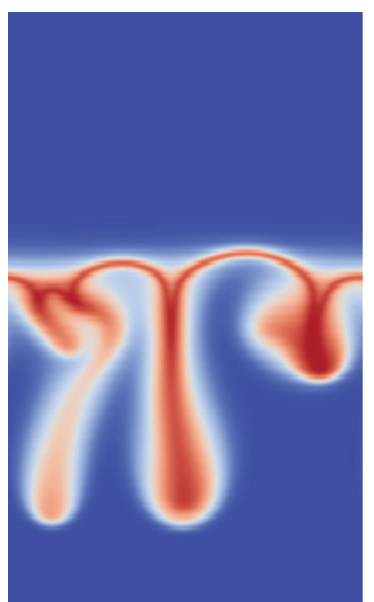

FIgURE 2: Concentration isosurfaces for $\alpha=0.0, G_{a}=G_{b}=1$, $G_{c}=4, R_{b}=0, R_{c}=-3, A_{r}=2, \mathrm{Pe}=1000$, and $D_{a}=1$.

$R_{c}=-3, G_{a}=G_{b}=1, G_{c}=4, A_{r}=2, \mathrm{Pe}=1000$, and $D_{a}=1$. These contours are virtually identical to those reported by Hejazi and Azaiez [24] for this set of parameters' values.

Furthermore, the numerical convergence of the solution was analysed by considering cases with different spatial resolutions that varied from $128 \times 128$ to $512 \times 512$ while varying the time step accordingly. It was found that a spatial resolution of $256 \times 256$ resulted in finger structures similar to those obtained with larger number of grid points. As a result, a spatial resolution of $256 \times 256$ with at time step $\Delta t=0.005$ is used to present the nonlinear simulation results for the effect of reversibility on the instability of the displacement process.

3.2. Parameters of the Study. In what follows, concentration isosurfaces are presented to examine the development of the instability and analyze the role of the chemical reversibility. The flow evolution will depend on both viscosities and densities mismatch between the chemical species, in addition to $\mathrm{Pe}, A_{r}, D_{a}$, and $D_{r}$. In order to limit the analysis to the effects of the reversibility of the chemical reaction, the following parameters are fixed as $A_{r}=2, \mathrm{Pe}=1000$, and $D_{a}=1$. Furthermore, the analysis will first examine the cases where instability is only driven by densities mismatch $\left(R_{b}=R_{c}=0\right)$ for both scenarios involving stable $\left(G_{a}<\right.$ $\left.G_{b}\right)$ and unstable $\left(G_{a}>G_{b}\right)$ initial fronts. The effects of mobility mismatch are examined afterwards. For each case, concentration isosurfaces of the product $(C)$ are presented for a complete chemical reaction $(\alpha=0)$ and for one or two different nonzero values of the reversibility coefficient $(\alpha)$ in order to illustrate the effect of reversibility on the dynamics of fingering.

3.3. Effect of the Densities Mismatch at Equal Mobility Ratios. In this part, it is assumed that there is no viscosity mismatch between the chemical species $\left(R_{b}=R_{c}=0\right)$ and therefore the frontal instability depends only on the variation of densities between the reactants and the product. Flows with unstable initial reactive fronts $\left(G_{a}>G_{b}\right)$ are presented first followed by cases with stable initial fronts $\left(G_{a}<G_{b}\right)$.

3.3.1. Unstable Initial Reactive Front $\left(G_{a}>G_{b}\right)$. In a vertical displacement process, the initial interface between the displacing and displaced fluids is unstable if the top fluid $(A)$ is heavier than the bottom one $(B)\left(G_{a}>G_{b}\right)$. The density of the chemical product $(C)$ can be either smaller than, larger than, or in between the densities of $(A)$ and $(B)$. As a result, regardless of the density of product $(C)$, instability will take place at least at the trailing or leading fronts, if not at both. In what follows, various cases of instability are discussed.

The case where the density of the product $(C)$ is larger than those of both reactants, that is, $G_{c}>G_{a}>G_{b}$, resulted in a stable trailing front and an unstable leading front. As a result, fingers appeared and extended in the direction of the flow on the leading front as depicted in Figure 3. The figure shows results for the cases where the chemical reaction is complete $(\alpha=0)$, weakly reversible $(\alpha=0.3)$, and strongly reversible $(\alpha=0.8)$.

It is clear that, in this case, reversibility tends to attenuate the instability of the flow at the unstable leading front, while there was a noticeable increase in the instability at the trailing front. In this particular case $\left(G_{a}=4 ; G_{b}=1 ; G_{c}=10\right)$, it was found that the number of developed fingers increases as the reaction reverses. However, fingers are less developed and more diffuse than in the nonreversible case. Furthermore, for the reversible cases $(\alpha>0)$, there is a noticeable variation in the number of fingers as well as their structure as the magnitude of $\alpha$ changes.

It is worth mentioning that the effects of reversibility on the instability may vary based on the particular choice of density differences between the chemical species. To illustrate this, another simulation was carried out for the previous case of frontal instability, where instability still takes place at the leading front but uses different magnitudes of the densities $\left(G_{a}=2 ; G_{b}=1 ; G_{c}=4\right)$. In this case, the densities mismatch at the initial interface between the reactants and that at the unstable leading front are smaller compared to the previous case $\left(G_{a}=4 ; G_{b}=1 ; G_{c}=10\right)$ and the results for both complete and reversible reactions are presented in Figure 4. It can be noticed that reversibility still attenuates the instability of the system. However, changes in the magnitude of reversibility have now virtually no effect on both the development and structures of fingers.

When the density of the product $(C)$ is smaller than those of both reactants, the variation of densities is favorable at the trailing front $\left(G_{c}<G_{b}\right)$ and unfavorable at the leading one $\left(G_{a}>G_{c}\right)$. This combination of densities mismatches triggers instability at the trailing front, while the leading one will be stable. A simulation was carried out for $\left(G_{a}=4 ; G_{b}=2 ; G_{c}=\right.$ 1) and it was found that here too reversibility acts towards reducing the growth of fingers and results in more diffuse, less developed fingers. In addition, similar finger structures were observed for reversible reactions with different $\alpha$ as the variation in densities decreases $\left(G_{a}=3 ; G_{b}=2 ; G_{c}=\right.$ 1). However, for brevity the corresponding contours are not shown. 


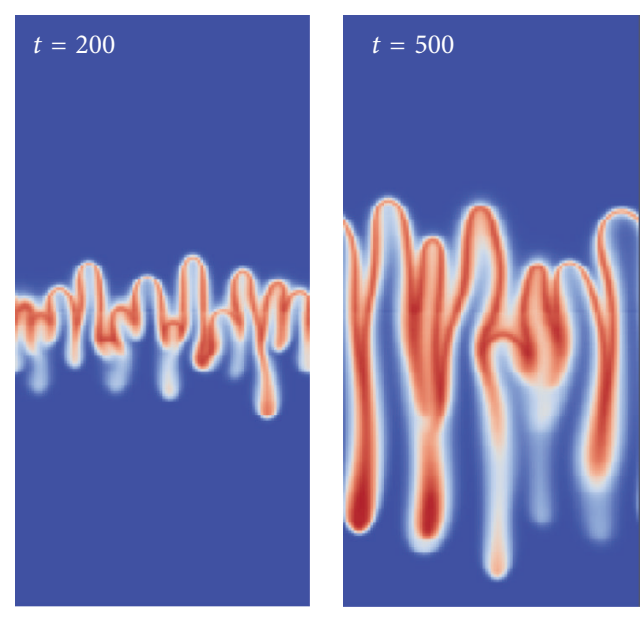

(a)

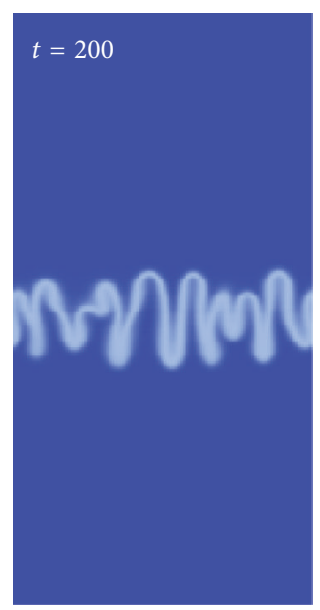

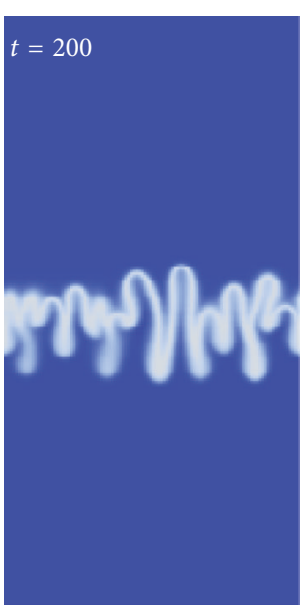

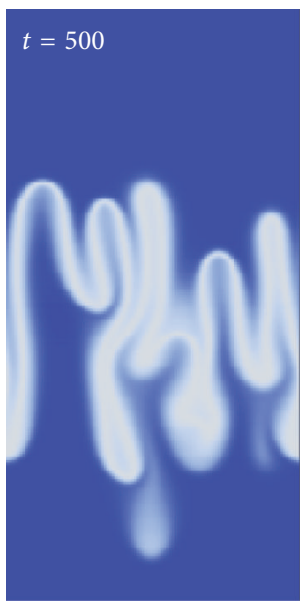

(b)

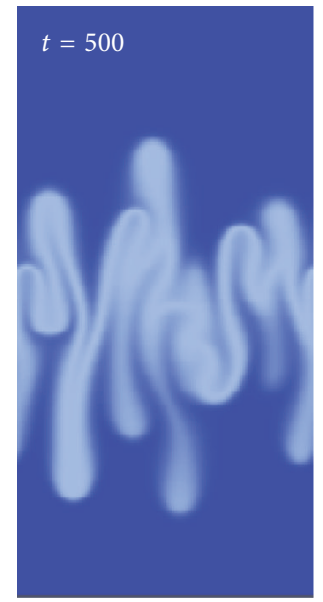

(c)

Figure 3: Concentration isosurfaces for $G_{a}=4, G_{b}=1$, and $G_{c}=10$ (stable trailing front, unstable leading front): (a) $\alpha=0.0$, (b) $\alpha=0.3$, and (c) $\alpha=0.8$.

In a reactive displacement process, instability will not grow at the unstable front until a certain amount of product (C) is produced. Furthermore, for cases with an unstable initial front, where instability appears at only one of the trailing or the leading fronts the densities mismatch at the unstable front is always larger than that of the initial interface. As the reaction reverses and part of the chemical product $(C)$ is converted to $(A)$ and $(B)$, the variation of densities between the reactants will attenuate the instability by decreasing the density differences at the unstable trailing or leading front. Furthermore, the presence of a stable front limits the degree of mixing between the reactants and this, in addition to the reduction in the amount of chemical product, attenuates the instability of the flow. This also explains the fact that there were no noticeable effects on the fingers development as the magnitude of the reversibility is changed in the case where a small variation between the densities mismatches at the initial and the unstable leading fronts is considered $\left(G_{a}=2 ; G_{b}=1\right.$; $\left.G_{c}=4\right)$.

For displacements where the density of the product $(C)$ lies between those of the two reactants, both the trailing and leading fronts are unstable $\left(G_{c}>G_{b}, G_{c}<G_{a}\right)$ as shown in Figure 5 for $G_{a}=3, G_{b}=1$, and $G_{c}=2$. In this particular case, it can be noticed that reversibility has a small tendency to attenuate the instability of the system. The rather limited effect of the chemical reversibility in such a system is due to the rapid mixing between the reactants as instability takes place at both the trailing and the leading fronts. In addition, the variations between densities at both fronts are less than that of the initial interface and as the reaction reverses, the favorable densities mismatch between the reactants helps to keep the instability growing, regardless of how fast product $(C)$ is converted back to $(A)$ and $(B)$. It should be noted that, in this particular case, stronger reversibility has a hardly noticeable effect on the structure of the fingers.

In all previous cases, reversibility tends to attenuate or slightly enhance the instability of the reactive system. However, the effect of reversibility on the instability where both the trailing and the leading fronts are unstable may vary as the densities gap between the reactants increases. This is well illustrated in Figure 6 where $G_{a}=4, G_{b}=1$, and $G_{c}=2$. For this choice of the density coefficients, there is actually 


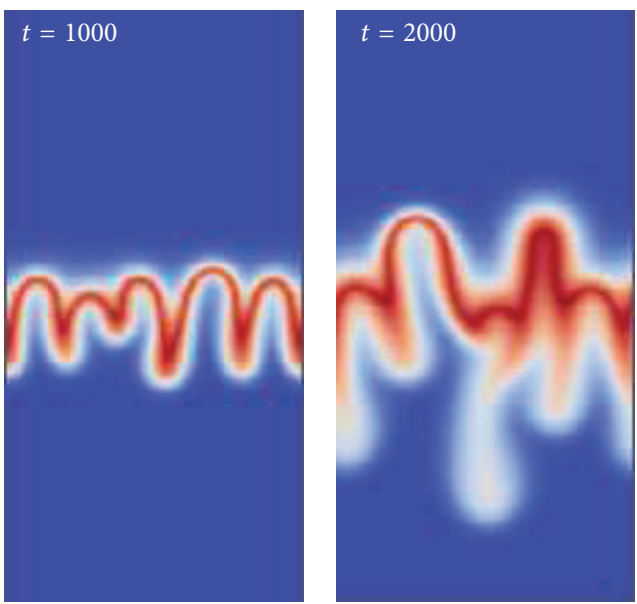

(a)

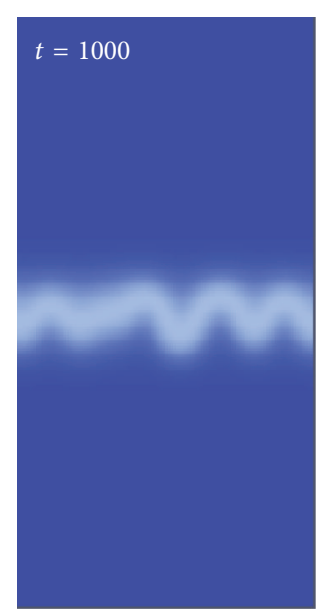

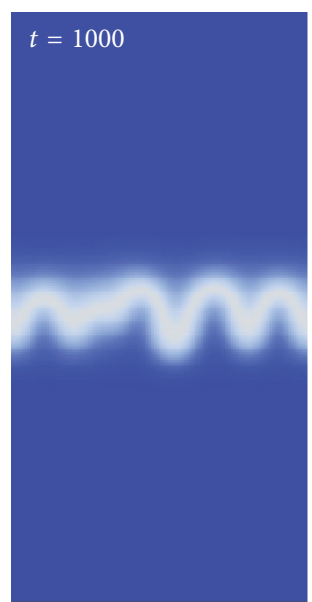

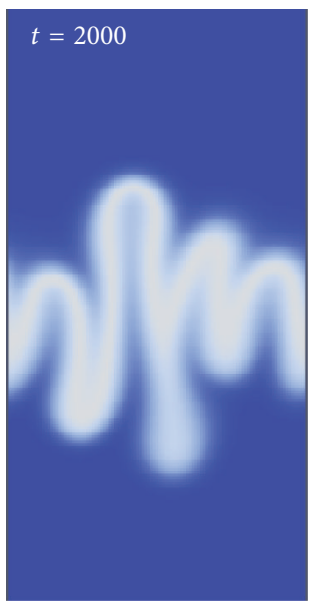

(b)

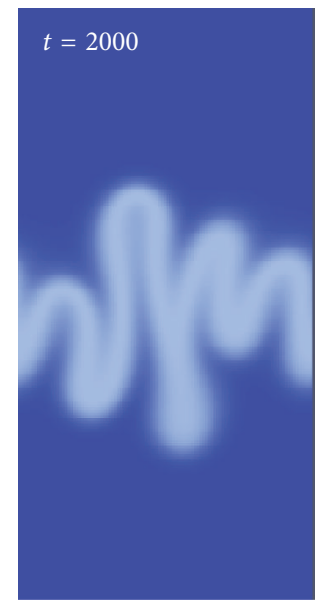

(c)

Figure 4: Concentration isosurfaces for $G_{a}=2, G_{b}=1$, and $G_{c}=4$ (stable trailing front, unstable leading front): (a) $\alpha=0.0$, (b) $\alpha=0.3$, and (c) $\alpha=0.8$.

a tendency for the fingers at both the trailing and leading fronts to extend more and have more interactions, when the reaction is reversible. Here, the fingers are more diffuse but more developed in the reversible cases, and the change in the degree of reversibility (i.e., $\alpha$ ) does actually have a strong noticeable effect on the fingers structures.

The strong effect of reversibility in increasing the instability at the leading front can be explained by looking at the variation of the density coefficients at that interface. When the chemical reaction is complete, the growth of instability at the leading front depends on the densities gap between reactant $(B)$ and product $(C)\left(G_{b}=1 ; G_{c}=2\right)$. However, as the reaction reverses, the favorable densities mismatch of the initial interface $\left(G_{a}=4 ; G_{b}=1\right)$ increases the variation in densities mismatch at the leading front, which explains the effect of reversibility in boosting the instability at that front. On the other hand, the small variation in densities gaps at the initial and the trailing fronts explains the limited effect of reversibility on the instability at the trailing front.
3.3.2. Stable or Neutrally Stable Initial Reactive Front $\left(G_{a} \leq\right.$ $\left.G_{b}\right)$. The initial interface between the two reactants is stable or neutrally stable if the density of the displacing fluid is either smaller or equal to that of the displaced fluid $\left(G_{b} \geq\right.$ $\left.G_{a}\right)$. However, as the reaction takes place and product $(C)$ appears at the initial interface, depending on the density of the product, instability may develop at either the trailing or the leading front, but not at both. The case where the density of $(C)$ lies between those of $(B)$ and $(A)$ or is equal to both or any of them $\left(G_{b} \geq G_{c} \geq G_{a}\right)$ will result in a stable displacement process and therefore will not be discussed. In what follows, the two cases where instability appears at either the trailing or the leading front are discussed.

An unstable trailing front and a stable leading front are observed in the case where the density of chemical product is smaller than that of both reactants. On the other hand, an unstable leading front and a stable trailing front are observed when the density of the product $(C)$ is larger than those of both reactants $\left(G_{a}=1 ; G_{b}=2 ; G_{c}=5\right)$ as shown in Figure 7. It is worth mentioning that unlike the 


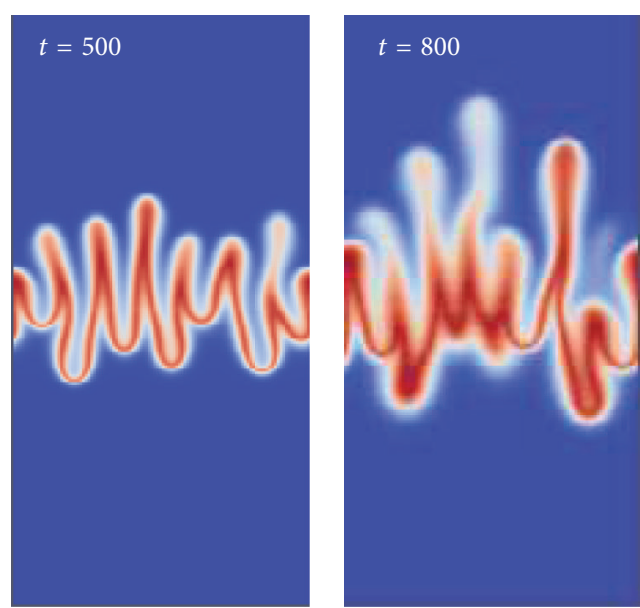

(a)

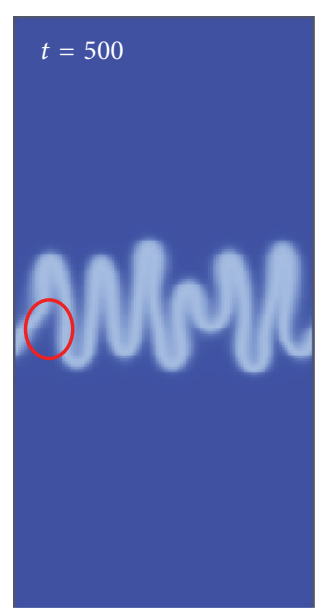

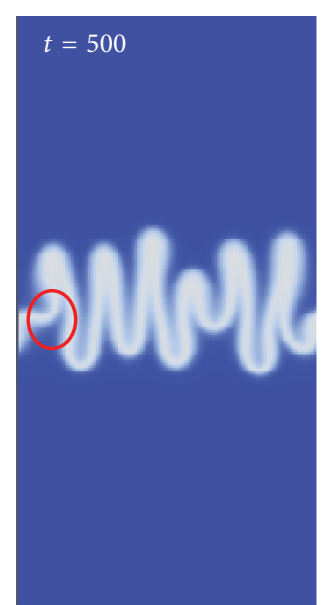

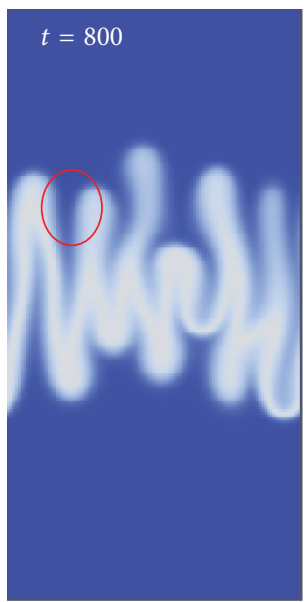

(b)

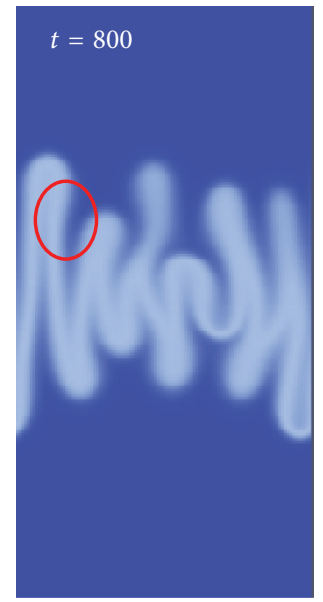

(c)

Figure 5: Concentration isosurfaces for $G_{a}=3, G_{b}=1$, and $G_{c}=2$ (unstable trailing front, unstable leading front): (a) $\alpha=0.0,(\mathrm{~b}) \alpha=0.3$, and (c) $\alpha=0.8$.

previously discussed cases with an unstable initial front the mixing between the two reactants when the initial front is stable is mainly controlled by diffusion. As a result, the growth of fingers is rather slow compared to cases with an unstable initial front. In both cases, reversibility tends to reduce the instability at the unstable front and may actually result in a stable system for a period of time. This reduction in instability is due to the fact that the unfavorable densities gap between the reactants reduces the variation of densities at the unstable front. However, depending on the magnitude of the reversibility coefficient and the variation of densities at the unstable front, instability will eventually develop at the unstable front at later times. A small reversibility coefficient combined with a large densities gap will result in an earlier development of the instability as shown in Figure 8. A complete stabilization of the system in these cases is due to the limited amount of the product $(C)$ at the unstable front, which in addition to the very weak mixing between the reactants delayed the development of the instability. The case where the instability takes place at the trailing front $\left(G_{a}=2 ; G_{b}=4\right.$; $\left.G_{c}=1\right)$ was also examined. Here too, reversibility attenuated the instability and resulted in stable system for a period of time; however for brevity the corresponding contours are not presented.

3.4. Effect of the Mobility Ratios. In a displacement process, the variations in the densities of the fluids involved are not the only source of instability. In fact, frontal instability can also be encountered as the viscosities of these fluids vary. In a displacement process, the interface between the displacing and displaced fluid is unstable or stable, if the viscosity of the displacing fluid is smaller or larger than that of the displaced one, respectively. In this part, the effect of viscosities mismatch on the instability of a reversible reactive displacement process is examined by including the variations of the viscosities of the species involved in the displacement in addition to their densities.

In a vertical displacement process, a stable or neutrally stable viscous interface $\left(R_{b} \leq 0\right)$ will limit the degree of mixing between the chemical species and consequently attenuates the instability of the system. Moreover, a favorable mobility ratio $\left(R_{b}>0\right)$ at the initial interface will help the 


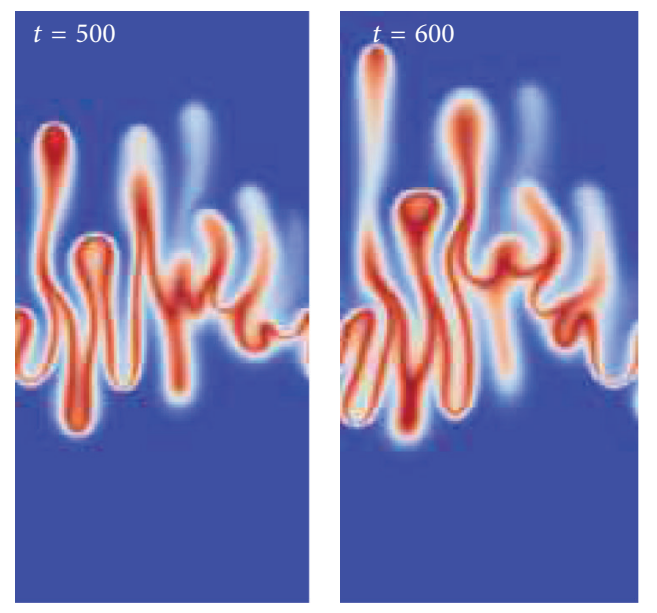

(a)

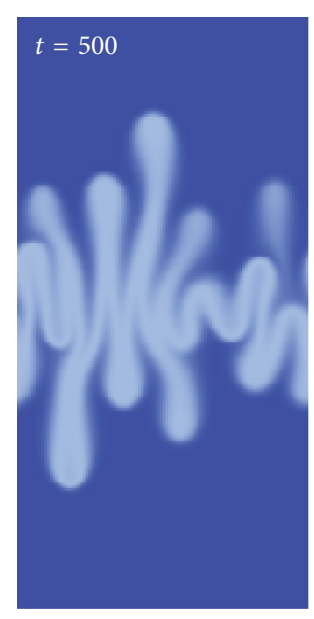

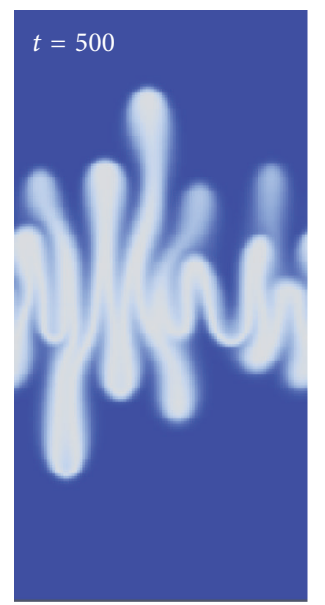

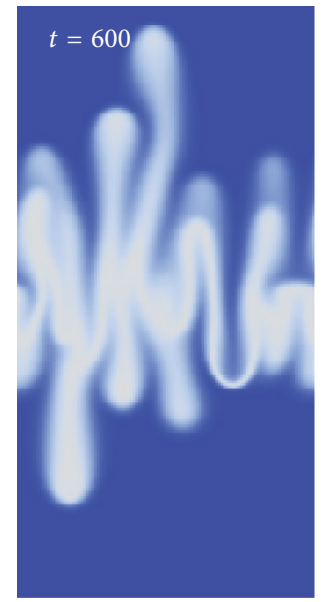

(b)

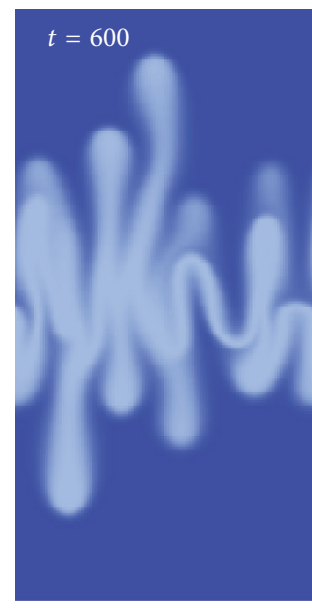

(c)

FIGURE 6: Concentration isosurfaces for $G_{a}=4, G_{b}=1$, and $G_{c}=2$ (unstable trailing front, unstable leading front): (a) $\alpha=0.0$, (b) $\alpha=0.3$, and (c) $\alpha=0.8$.

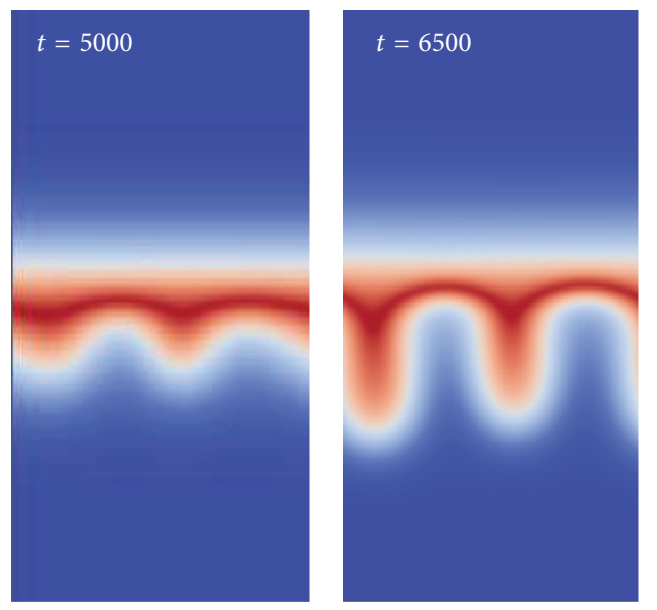

(a)

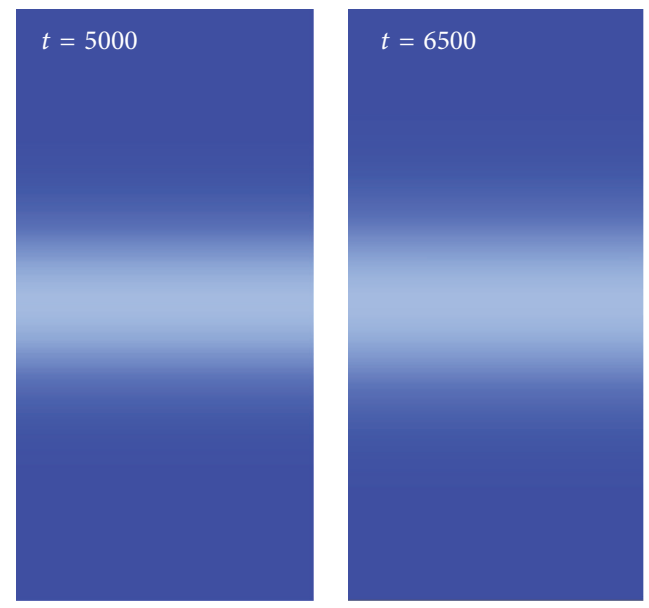

(b)

FIGURE 7: Concentration isosurfaces for $G_{a}=1, G_{b}=2$, and $G_{c}=5$ (stable trailing front, unstable leading front): (a) $\alpha=0.0$, (b) $\alpha=0.8$. 


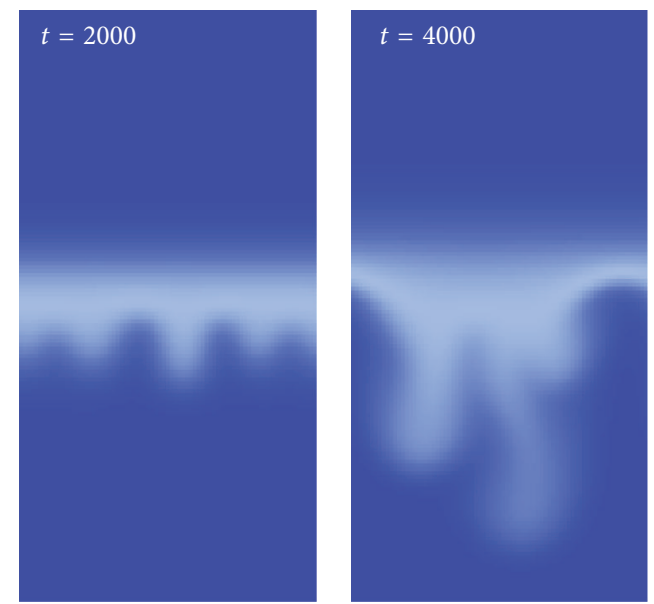

FIGURE 8: Concentration isosurfaces for $G_{a}=1, G_{b}=2, G_{c}=10$, and $\alpha=0.8$ (stable trailing front, unstable leading front).

mixing between the species and enhance the instability of the system. However, in the absence of injection, the mixing between the species is controlled by diffusion rather than convection.

Starting with the unstable initial viscous interface, mobility ratios of $R_{b}=2$ and $R_{c}=3$, which result in both the trailing $\left(R_{c} / 2\right)$ and the leading $\left(R_{b}-R_{c} / 2\right)$ fronts being viscously unstable. Figure 9 depicts the results for the examined case for both complete $(\alpha=0)$ and strongly reversible $(\alpha=0.8)$ reactions. From the figure, it can be noticed that the growth of the instability is much weaker compared to the same case in the absence of viscosities' contrast (Figure 6).

The case where the viscous initial interface between the reactants is stable $\left(R_{b}<0\right)$ is examined using mobility ratios of $R_{b}=-2$ and $R_{c}=3$. Here, the trailing front is viscously unstable $\left(R_{c} / 2\right)$, while the leading front is viscously stable. The nonlinear simulation results are presented in Figure 10 for both cases of complete $(\alpha=0)$ and extremely reversible $(\alpha=0.8)$ reactions. In this case, the unfavorable mobility ratio between the reactants enhances the mixing between the chemical species, which enriched the instability of the system and resulted in an earlier development of the instability compared to the same case in absence of viscosities mismatch (Figure 4).

It is worth mentioning that, in the previous two cases, reversibility had an opposite influence on the instability where it enhanced/attenuated the instability instead of attenuating/enhancing the instability for the cases $\left(G_{a}=2 ; G_{b}=1\right.$; $\left.G_{c}=4\right)$ and $\left(G_{a}=4 ; G_{b}=1 ; G_{c}=2\right)$, respectively. Furthermore, the previous two cases were considered as an example where either the densities or viscosities mismatch was in favor of the growth of the instability while the other was not. However the instability will be enhanced/attenuated when both densities and viscosities mismatches are in favor of the growth/reduction of the instability. Finally, it should be stressed that these results are observed in the absence of injection and the effects of viscosities on the instability may actually vary with the speed of injection. The observed effects of the variation of viscosities can be explained by the countered relation between viscosity and diffusion $[9$, 36]. In the absence of injection, the mixing is controlled by diffusion and the increased/decreased mobility ratio between the reactants limits/helps the mixing between the reactants and as a consequence attenuates/enhances the instability.

3.5. Quantitative Analysis. In this part, the effects of reversibility on the instability of the reactive system are illustrated by presenting the relative contact area (R.C.A.) of the system, which is defined as the area of contact between the species involved in the displacement process scaled by the cross-sectional area of the cell.

This contact area is determined by measuring the length of a contour that corresponds to a specific concentration's value of one of the species, which is the product $(C)$ in this case. There are two contact interfaces to be measured; one corresponds to the leading front and the other to the trailing front. The contour that corresponds to concentration of 0.01 of the chemical product $(C=0.01)$ is used to determine the length of the contact areas at both fronts.

Measuring the contact area between the species is a good criterion to quantify the development of the instability. Moreover, this contact area is expected to increase as the complexity of the fingering structures increases. The R.C.A. for the case where an increase in the instability of the reactive front was observed $\left(G_{a}=4 ; G_{b}=1 ; G_{c}=2\right)$ has been determined and its variation with time is depicted in Figure 11. In this case, the densities mismatch triggers instability at both fronts in the absence of viscous effects. The results confirm what has been reported in the qualitative part where an increase in the instability of the leading front has been observed as the reaction reverses. Furthermore, it can be noticed that the growth of the instability or equivalently the contact area between the species at the trailing front is larger than that at the leading one, which is a direct consequence of the larger densities mismatch at the trailing front.

Furthermore, the relative contact areas were also determined for the cases where the instability is triggered by both density and viscosity mismatches and it was observed that the contact areas grow faster/slower than the cases with only density mismatch, as the viscosity mismatches help/attenuate the instability when the reaction reverses.

It is worth mentioning that the growth of the instability when the reaction reverses is always slower than that of the complete reactions, at both the trailing and the leading fronts at early stages. Again, this is a result of the reduction in the total amount of the chemical product $(C)$. However, as time proceeds and more chemical product accumulates at the reactive interfaces, the effect of converting part of $(C)$ back to $(A)$ and $(B)$ becomes more pronounced.

\section{Conclusion and Discussion}

The main objective of this study was to determine the effects of chemical reversibility on the overall efficiency of a vertical reversible reactive displacement process. The frontal instability is triggered by the densities combined or not with 


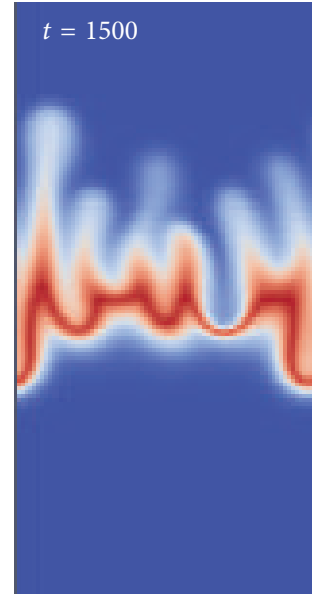

(a)

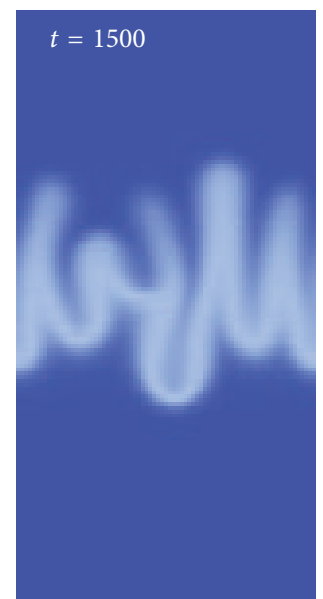

(b)

Figure 9: Concentration isosurfaces for $G_{a}=4, G_{b}=1, G_{c}=2, R_{b}=2$, and $R_{c}=3:$ (a) $\alpha=0.0$, (b) $\alpha=0.8$.

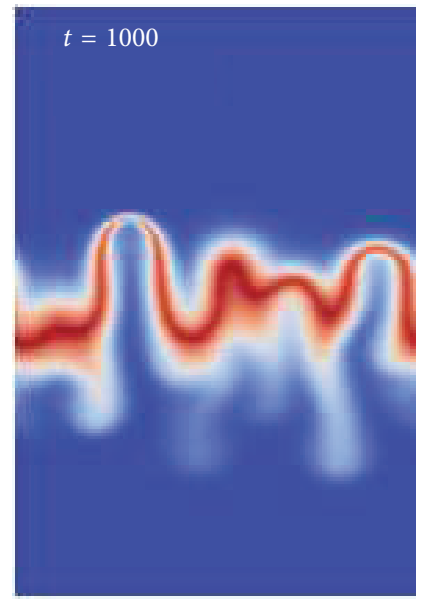

(a)

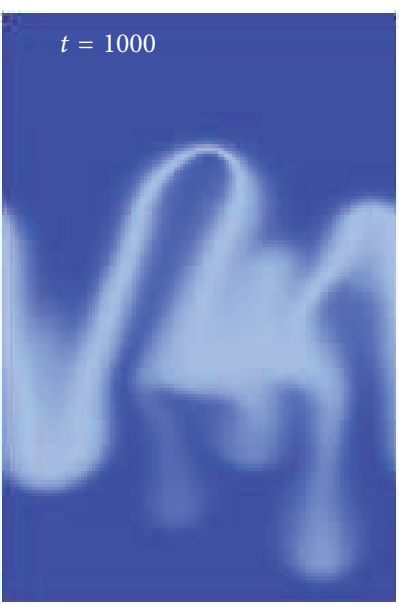

(b)

FIGURE 10: Concentration isosurfaces for $G_{a}=2, G_{b}=1, G_{c}=4, R_{b}=-2$, and $R_{c}=3$ : (a) $\alpha=0.0$, (b) $\alpha=0.8$.

viscosities' mismatch between the reactants and the product. The nonlinear interactions between the fluids were captured for different scenarios of frontal instability at specific sets of parameters. It was found that the fate of the displacement process can be dramatically influenced when a reversible chemical reaction takes place between the displacing and the displaced fluids, which introduces a new fluid with a viscosity and density that might be different from those of both reactants. The dynamics of fingering were mainly controlled by the viscosities and densities mismatch at the initial front as well as the trailing and the leading fronts in addition to the magnitude of the reversibility of the reaction.

The development of the instability was much faster in cases where the initial front between the reactants was unstable $\left(G_{a}>G_{b}\right)$ compared to cases with a stable initial front $\left(G_{a} \leq G_{b}\right)$. This was attributed to the higher production rate in the former case, which is a result of the fast mixing between the reactants. The frontal instability of the reactive leading and trailing fronts determines the direction of fingers development, where they develop in the upstream or downstream direction when the instability appears only at the trailing or the leading front, respectively, if not in both directions when both fronts are unstable.

In the absence of viscosity differences between the chemicals, the effect of chemical reversibility was found to be similar for cases where only one of the trailing or the leading fronts is unstable regardless of the nature of the instability at the initial front. For those cases, the attenuation of the fingering instability increases with the increase in the magnitude of reversibility. Moreover, a complete stabilization of the system was reported for cases with stable initial fronts $\left(G_{a} \leq G_{b}\right)$. Furthermore, for unstable initial fronts similar fingering structures were observed for reversible reactions with different magnitude of reversibility as the densities difference between the reactants decreases. It was also found that reversibility has a weak tendency to attenuate the instability 


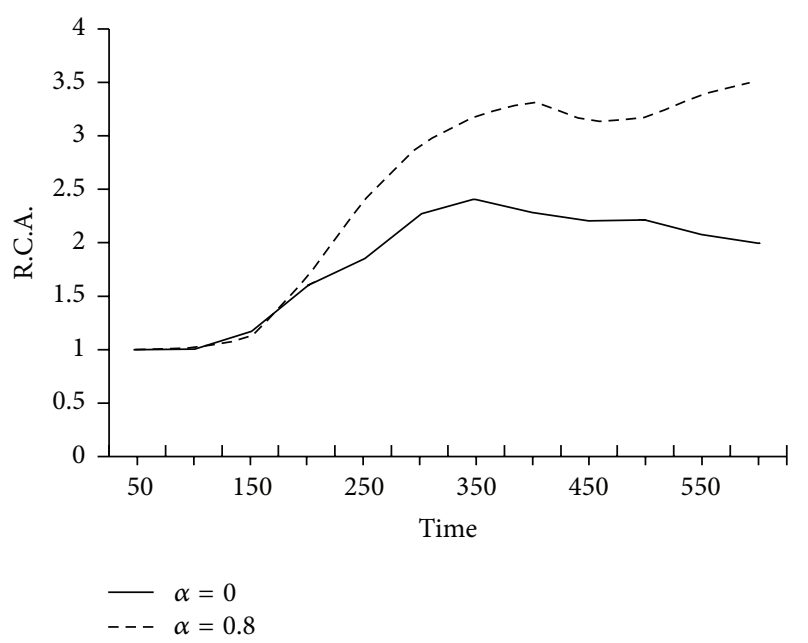

(a)

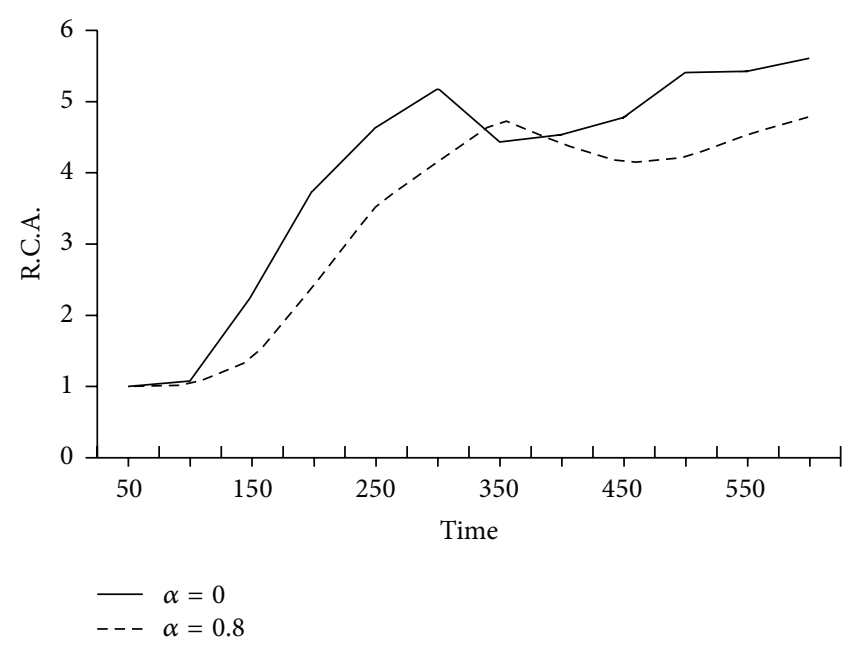

(b)

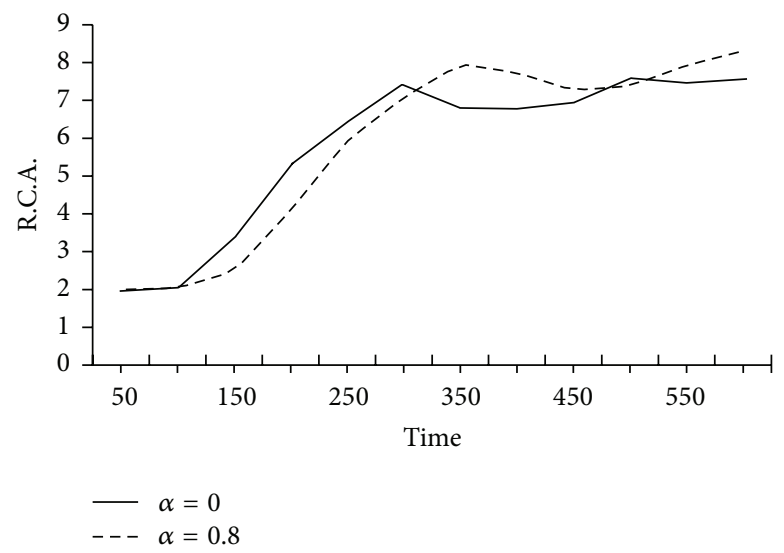

(c)

FIgURE 11: Variations of the relative contact area (R.C.A.) for $G_{a}=4, G_{b}=1, G_{c}=2$, and $R_{b}=R_{c}=0$ : (a) R.C.A. at the leading front, (b) R.C.A. at the trailing front, and (c) total R.C.A.

when both of the reactive fronts are unstable combined with virtually unnoticeable changes in the fingering structures when there is a small variation in the densities $\left(G_{a}=3 ; G_{b}=\right.$ $1 ; G_{c}=2$ ). However, an enhancement of the instability was observed as the variation of the densities increases $\left(G_{a}=4\right.$; $G_{b}=1 ; G_{c}=2$ ). The fast mixing between the reactants and the favorable densities mismatch between the reactants help sustaining the growth of the instability regardless of the magnitude of the reversibility. These results indicate that the general overall stabilizing effects of chemical reversibility would lead to a smoother front and consequently a better sweep efficiency of the process. The more diffuse nature of the interface implies stronger mixing of the displaced and displacing fluid.

Finally, it was observed that increasing the viscosities mismatch between the chemical species in order to achieve viscously unstable trailing and leading fronts attenuated the instability of the system by limiting the degree of mixing between the chemicals. On the other hand, the stable initial viscous interface $\left(R_{b}<0\right)$ enhanced the instability of the displacement process.

\section{Conflict of Interests}

The authors declare that there is no conflict of interests regarding the publication of this paper.

\section{Acknowledgments}

H. Alhumade acknowledges financial support from the Ministry of Education, Kingdom of Saudi Arabia. The use of computational resources from WestGrid is also acknowledged.

\section{References}

[1] S. Hill, "Channeling in packed columns," Chemical Engineering Science, vol. 1, no. 6, pp. 247-253, 1952.

[2] R. L. Slobod and B. H. Caudle, "X-ray shadowgraph studies of areal sweep-out efficiencies," Transactions of the American Institute of Mining and Metallurgical Engineers, vol. 195, pp. 265270, 1952.

[3] P. G. Saffman and G. Taylor, "The penetration of a fluid into a porous medium or Hele-Shaw cell containing a more viscous 
liquid," Proceedings of the Royal Society. London. Series A. Mathematical, Physical and Engineering Sciences, vol. 245, no. 1242, pp. 312-329, 1958.

[4] G. M. Homsy, "Viscous fingering in porous media," Annual Review of Fluid Mechanics, vol. 19, pp. 271-311, 1987.

[5] K. V. McCloud and J. V. Maher, "Experimental perturbations to Saffman-Taylor flow," Physics Report, vol. 260, no. 3, pp. 139-185, 1995.

[6] C. Bacri, D. Salin, and Y. Yortsos, "Analyse linéaire de la stabilité de l'écoulement de fluides miscibles en milieux poreux," Comptes Rendus de l'Académie des Sciences-Paris, vol. 314, pp. 139-144, 1992.

[7] A. Rogerson and E. Meiburg, "Shear stabilization of miscible displacement processes in porous media," Physics of Fluids A, vol. 5, no. 6, pp. 1344-1355, 1992.

[8] A. Rogerson and E. Meiburg, "Numerical simulation of miscible displacement processes in porous media flows under gravity," Physics of Fluids A, vol. 5, no. 11, pp. 2644-2660, 1993.

[9] O. Manickam and G. M. Homsy, "Fingering instabilities in vertical miscible displacement flows in porous media," Journal of Fluid Mechanics, vol. 288, pp. 75-102, 1995.

[10] J. Al-Humoud and A. J. Chamkha, "Reactive contaminant transport with space-dependent dispersion and time-dependent concentration source," Journal of Porous Media, vol. 10, no. 4, pp. 377-390, 2007.

[11] A. Yarahmadi, R. Hosseini, and M. R. H. Nobari, "Numerical inspection of turbulent/laminar nonreactive andlaminar combustive reactive flows in porous media," Journal of Porous Media, vol. 13, no. 6, pp. 511-528, 2010.

[12] A. Bousri, K. Bouhadef, H. Beji, R. Bennacer, and R. Nebbali, "Heat and mass transfer in reactive porous media with local nonequilibrium conditions," Journal of Porous Media, vol. 15, no. 4, pp. 329-341, 2012.

[13] A. Marafie, "Modeling of flow through a reactive porous plug as related to biological applications," Journal of Porous Media, vol. 15, no. 9, pp. 823-833, 2012.

[14] D. Srinivasacharya and G. S. Reddy, "Free convection in a non-newtonian power-lawfluid-saturated porous medium with chemical reaction and radiation effects," Special Topics and Reviews in Porous Media, vol. 4, no. 3, pp. 223-236, 2013.

[15] T. Podgorski, M. C. Sostarecz, S. Zorman, and A. Belmonte, "Fingering instabilities of a reactive micellar interface," Physical Review E, vol. 76, no. 1, Article ID 016202, 2007.

[16] Y. Nagatsu, K. Matsuda, Y. Kato, and Y. Tada, "Experimental study on miscible viscous fingering involving viscosity changes induced by variations in chemical species concentrations due to chemical reactions," Journal of Fluid Mechanics, vol. 571, pp. 475-493, 2007.

[17] Y. Nagatsu, Y. Kondo, Y. Kato, and Y. Tada, "Effects of moderate Damköhler number on miscible viscous fingering involving viscosity decrease due to a chemical reaction," Journal of Fluid Mechanics, vol. 625, pp. 97-124, 2009.

[18] Y. Nagatsu, Y. Kondo, Y. Kato, and Y. Tada, "Miscible viscous fingering involving viscosity increase by a chemical reaction with moderate Damköhler number," Physics of Fluids, vol. 23, no. 1, Article ID 014109, 2011.

[19] L. Rongy, P. M. J. Trevelyan, and A. De Wit, "Dynamics of $A+B \rightarrow C$ reaction fronts in the presence of buoyancy-driven convection," Physical Review Letters, vol. 101, no. 8, Article ID 084503, 2008.
[20] C. Almarcha, P. M. J. Trevelyan, P. Grosfils, and A. De Wit, "Chemically driven hydrodynamic instabilities," Physical Review Letters, vol. 104, no. 4, Article ID 044501, 2010.

[21] H. Alhumade and J. Azaiez, "Stability analysis of reversible reactive flow displacements in porous media," Chemical Engineering Science, vol. 101, pp. 46-55, 2013.

[22] H. Alhumade and J. Azaiez, "Numerical simulations of reversible reactive flows in homogeneous porous media," Journal of Porous Media, vol. 17, no. 4, pp. 359-372, 2014.

[23] S. H. Hejazi and J. Azaiez, "Stability of reactive interfaces in saturated porous media under gravity in the presence of transverse flows," Journal of Fluid Mechanics, vol. 695, pp. 439466, 2012.

[24] S. H. Hejazi and J. Azaiez, "Nonlinear simulation of transverse flow interactions with chemically driven convective mixing in porous media," Water Resources Research, vol. 49, no. 8, pp. 4607-4618, 2013.

[25] Q. Yuan and J. Azaiez, "Cyclic time-dependent reactive flow displacements in porous media," Chemical Engineering Science, vol. 109, pp. 136-146, 2014.

[26] T. C. Flowers and J. R. Hunt, "Viscous and gravitational contributions to mixing during vertical brine transport in watersaturated porous media," Water Resources Research, vol. 43, no. 1, Article ID W01407, 2007.

[27] J. Ennis-King and L. Paterson, "Coupling of geochemical reactions and convective mixing in the long-term geological storage of carbon dioxide," International Journal of Greenhouse Gas Control, vol. 1, no. 1, pp. 86-93, 2007.

[28] K. U. Mayer, E. O. Frind, and D. W. Blowes, "Multicomponent reactive transport modeling in variably saturated porous media using a generalized formulation for kinetically controlled reactions," Water Resources Research, vol. 38, no. 9, pp. 13-1-13-21, 2002.

[29] S. Grüner and A. Kienle, "Equilibrium theory and nonlinear waves for reactive distillation columns and chromatographic reactors," Chemical Engineering Science, vol. 59, no. 4, pp. 901918, 2004.

[30] M. N. Islam and J. Azaiez, "New viscous fingering mechanisms at high viscosity ratio and Péclet number miscible displacements," Journal of Porous Media, vol. 10, no. 4, pp. 357-376, 2007.

[31] T. Gérard and A. De Wit, "Miscible viscous fingering induced by a simple $\mathrm{A}+\mathrm{B} \rightarrow \mathrm{C}$ chemical reaction," Physical Review E-Statistical, Nonlinear, and Soft Matter Physics, vol. 79, no. 1, Article ID 016308, 2009.

[32] S. H. Hejazi and J. Azaiez, "Non-linear interactions of dynamic reactive interfaces in porous media," Chemical Engineering Science, vol. 65, no. 2, pp. 938-949, 2010.

[33] C. Canuto, M. Hussaini, A. Quarteroni, and T. Zang, Spectral Methods in Fluid Dynamic, Springer, New York, NY, USA, 1987.

[34] W. B. Zimmerman and G. M. Homsy, "Viscous fingering in miscible displacements: unification of effects of viscosity contrast, anisotropic dispersion, and velocity dependence of dispersion on nonlinear finger propagation," Physics of Fluids A, vol. 4, no. 11, pp. 2348-2359, 1992.

[35] M. N. Islam and J. Azaiez, "Fully implicit finite difference pseudo-spectral method for simulating high mobility-ratio miscible displacements," International Journal for Numerical Methods in Fluids, vol. 47, no. 2, pp. 161-183, 2005.

[36] I. Avramov, "Relationship between diffusion, self-diffusion and viscosity," Journal of Non-Crystalline Solids, vol. 355, no. 10-12, pp. $745-747,2009$. 


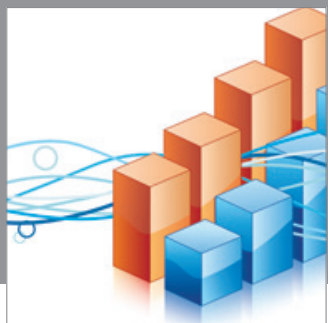

Advances in

Operations Research

mansans

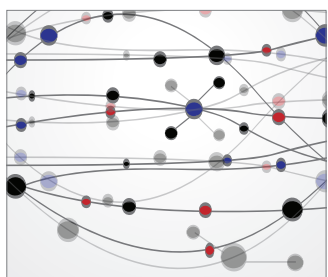

The Scientific World Journal
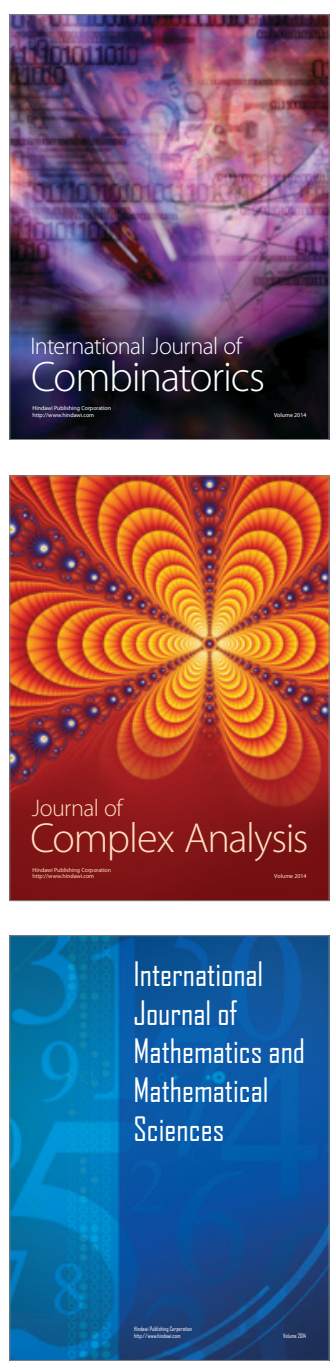
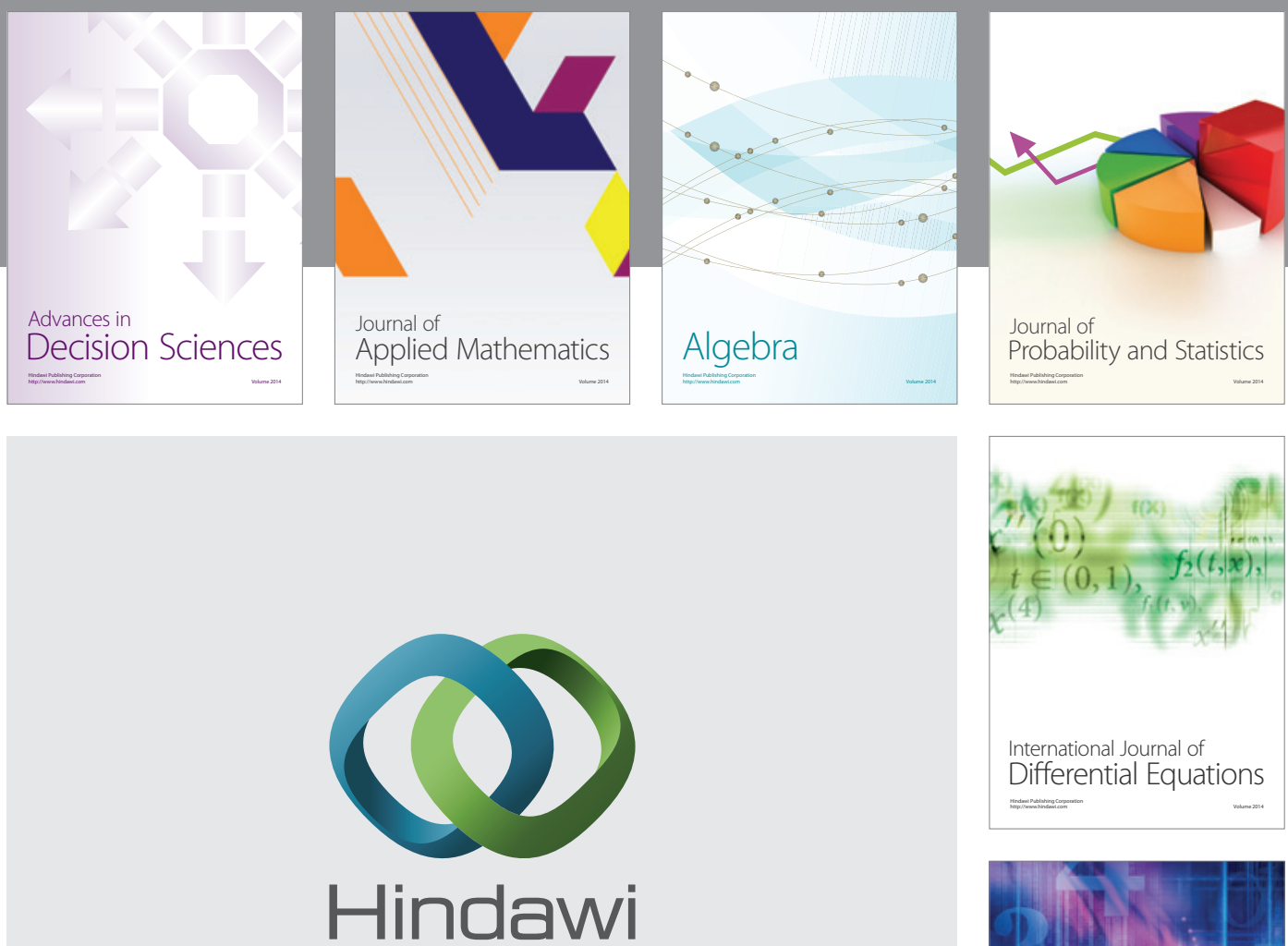

Submit your manuscripts at http://www.hindawi.com
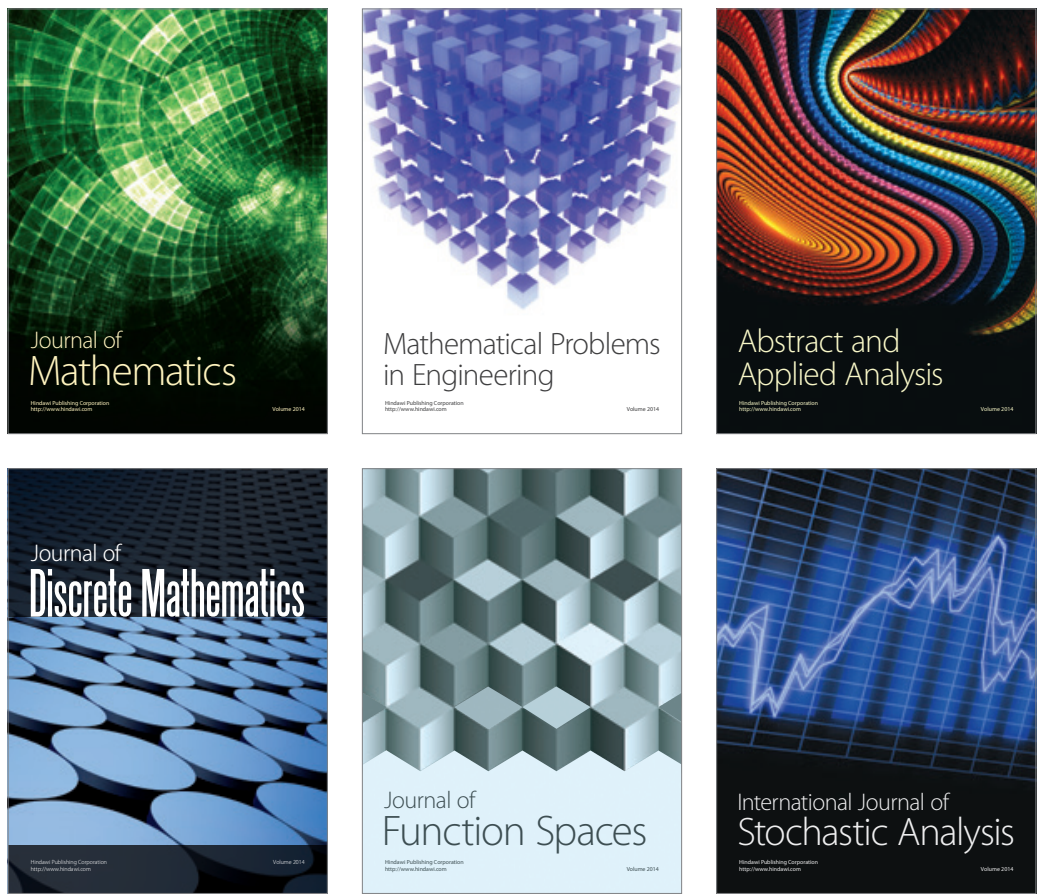

Journal of

Function Spaces

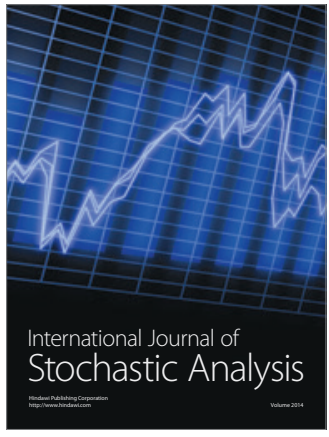

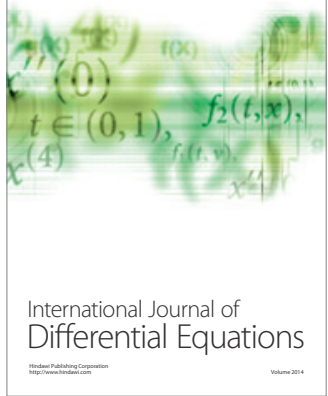
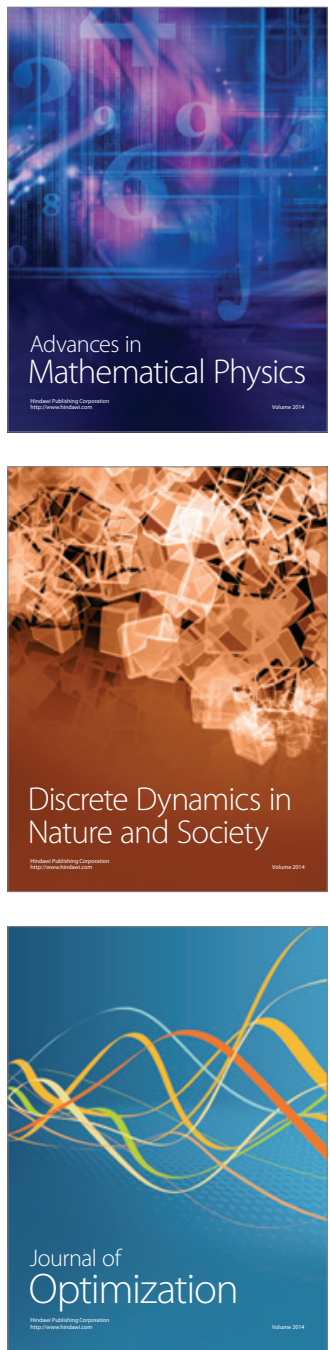